University of Nebraska - Lincoln

DigitalCommons@University of Nebraska - Lincoln

8-2008

\title{
Half-metallicity in hybrid BCN nanoribbons
}

\author{
Er-jun Kan \\ Hefei National Laboratory for Physical Sciences at Microscale, University of Science and Technology of \\ China, Hefei, Anhui 230026, People's Republic of China \\ Xiaojun Wu \\ University of Nebraska-Lincoln, xwu4@unl.edu \\ Zenyu Li \\ Hefei National Laboratory for Physical Sciences at Microscale, University of Science and Technology of \\ China, Hefei, Anhui 230026, People's Republic of China \\ Xiao Cheng Zeng \\ University of Nebraska-Lincoln, xzeng1@unl.edu \\ Jinlong Yang \\ Hefei National Laboratory for Physical Sciences at Microscale, University of Science and Technology of \\ China, Hefei, Anhui 230026, People's Republic of China \\ See next page for additional authors
}

Follow this and additional works at: https://digitalcommons.unl.edu/chemzeng

Part of the Chemistry Commons

Kan, Er-jun; Wu, Xiaojun; Li, Zenyu; Zeng, Xiao Cheng; Yang, Jinlong; and Hou, J. G., "Half-metallicity in hybrid BCN nanoribbons" (2008). Xiao Cheng Zeng Publications. 88.

https://digitalcommons.unl.edu/chemzeng/88

This Article is brought to you for free and open access by the Published Research - Department of Chemistry at DigitalCommons@University of Nebraska - Lincoln. It has been accepted for inclusion in Xiao Cheng Zeng Publications by an authorized administrator of DigitalCommons@University of Nebraska - Lincoln. 


\section{Authors}

Er-jun Kan, Xiaojun Wu, Zenyu Li, Xiao Cheng Zeng, Jinlong Yang, and J. G. Hou 


\title{
Half-metallicity in hybrid BCN nanoribbons
}

\author{
Er-Jun Kan, ${ }^{1}$ Xiaojun $\mathrm{Wu},{ }^{2}$ Zhenyu Li, ${ }^{1}$ X. C. Zeng, ${ }^{2}$ Jinlong Yang, ${ }^{1, a)}$ and J. G. Hou ${ }^{1}$ \\ ${ }^{1}$ Hefei National Laboratory for Physical Sciences at Microscale, \\ University of Science and Technology of China, Hefei, Anhui 230026, People's Republic of China \\ ${ }^{2}$ Department of Chemistry and Nebraska Center for Materials and Nanoscience, \\ University of Nebraska-Lincoln, Lincoln, Nebraska 68588, USA
}

(Received 16 June 2008; accepted 24 July 2008; published online 29 August 2008)

\begin{abstract}
The established chemical synthetic strategy toward graphene nanoribbons has greatly prompted and justified the research of theoretical designs of novel materials based on graphene. In this paper, we report the novel half-metallicity in $\mathrm{C}$ and $\mathrm{BN}$ hybrid zigzag nanoribbons even though stand-alone $\mathrm{C}$ or BN nanoribbon possesses a finite band gap. By performing first-principles electronic-structure calculations, we find this unexpected half-metallicity in the hybrid nanostructures stems from a competition between the charge and spin polarizations, as well as from the $\pi$ orbital hybridization between $\mathrm{C}$ and $\mathrm{BN}$. Molecular dynamics simulations indicate that the hybrid nanoribbons are stable. Our results point out a possibility of making spintronic devices solely based on nanoribbons and a new way of fabricating metal-free half metals. (C) 2008 American Institute of Physics.
\end{abstract}

[DOI: 10.1063/1.2971187]

\section{INTRODUCTION}

The discovery of low-dimensional carbon allotropes such as fullerenes ${ }^{1}$ and nanotubes ${ }^{2}$ has stimulated intensive research on metal-free magnetism, owing to their small spinorbit coupling and long spin scattering length. Since the first report of room-temperature weak ferromagnetism in polymerized $\mathrm{C}_{60},{ }^{3}$ magnetism in pure carbon materials has been demonstrated by several experimental groups. ${ }^{4-6}$ Carbon defects or adatoms are believed to be the origin for the unexpected magnetism. ${ }^{7-9}$

A question arises that can low-dimensional carbon nanostructures exhibit half-metallicity? Half metals are ideal for spintronic applications because they have one metallic spin channel and one semiconducting or insulating spin channel. Techniques for tuning electronic properties of graphene nanoribbon (GNR) have been advanced, ${ }^{10-12}$ for example, by terminating a single graphite layer in one direction. Recently, Son et al. ${ }^{13}$ predicted, based on density functional theory (DFT) calculation, that zigzag edge GNRs (ZGNRs) can be converted to half metal when an external transverse electric field is applied onto the graphene layer. However, theoretical studies have revealed that a very strong field is required to achieve half-metallicity. ${ }^{13-15}$

In the absence of external field, ZGNRs are semiconductor with two localized electronic edge states. ${ }^{15-24}$ These two ferromagnetically ordered edge states are antiferromagnetically coupled. In the presence of an external field, an electrostatic potential difference is generated between the two ZGNR edges, which causes band crossing and charge transfer between the two edge states. As a result, the ZGNRs are converted to half metal from semiconductor. Since the critical step to make half-metallic ZGNR is to break the symme-

\footnotetext{
a) Author to whom correspondence should be addressed. Electronic mail: jlyang@ustc.edu.cn.
}

try of the two edge states, an alternative approach is to use chemical edge modification. ${ }^{16}$ Obviously, for nanoelectronic applications, the chemical approach has many advantages over the strong-field approach.

In this paper, we present a theoretical design to achieve half-metallicity for carbon nanoribbons using structural hybridization. The designed materials are constructed by implanting a $\mathrm{BN}$ row into a ZGNR. The principle of the idea is based on the following observation: Both the highest valence band (VB) and the lowest conduction band (CB) of ZGNR are mainly contributed by the two edge states which have opposite spin but are degenerate in energy (as shown in Fig. 1). In contrast, for the zigzag $\mathrm{BN}$ nanoribbon, the highest VB is originated from the $\pi$ orbitals of the $\mathrm{N}$ edge atoms, while the lowest $\mathrm{CB}$ is mainly localized at the $\mathrm{B}$ edge atoms. These results suggest that the symmetry of the ZGNR band structure near the Fermi energy can be broken by introducing a segment of BN nanoribbon.

\section{MODELS AND METHODS}

The geometry of a hybrid C/BN nanoribbon is shown in Fig. 1(c), where the armchair atom rows are normal to the ribbon direction, while the zigzag atom chains are in the ribbon direction. At the two edges, $\mathrm{H}$ atoms are added to passivate the dangling $\sigma$ bonds. The hybrid-ribbon structures are denoted as $n-\mathrm{C}_{i} \mathrm{BN}$, where $i$ refers to the number of armchair $\mathrm{C}$ rows in a unit cell and $n$ is the width of the nanoribbon in term of the number of zigzag chains. On one edge, $\mathrm{B}$ is the outmost nonhydrogen atom; this edge is denoted as the B-edge. The outmost $\mathrm{C}$ atoms on the $\mathrm{B}$-edge side are named $C_{B}$. Similarly, the edge $C$ atoms on the $N$-edge side are named $\mathrm{C}_{\mathrm{N}}$.

The DFT calculations were carried out using the VASP package. $^{25}$ The projector augmented wave ${ }^{26}$ method in Kresse-Joubert implementation ${ }^{27}$ was used to describe the electron-ion interaction. The plane wave cutoff energy was 

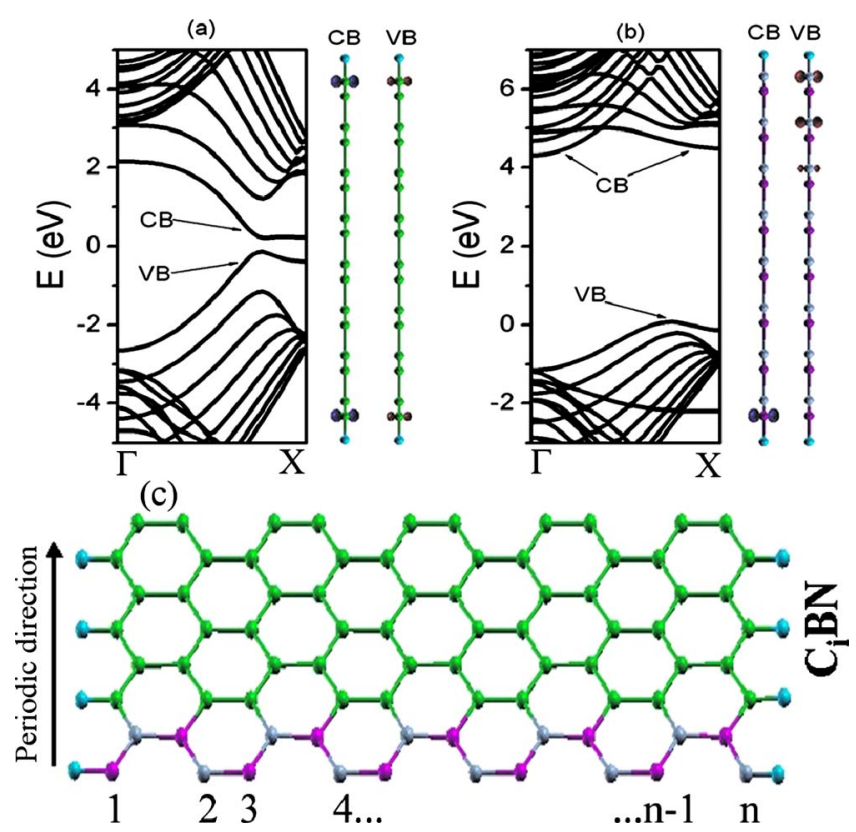

FIG. 1. (Color online) Band structures and band-resolved charge densities of (a) ZGNR and (b) BN nanoribbon with eight zigzag chains: Only the atom row including two edge atoms is shown in charge density plot. (c) The atomic configuration of $\mathrm{C}_{i} \mathrm{BN}$ ribbons. The green, pink, gray, and sapphire balls denote carbon, boron, nitrogen, and hydrogen atoms, respectively.

set to $400.0 \mathrm{eV}$. The convergence thresholds for energy and force were $10^{-5} \mathrm{eV}$ and $0.01 \mathrm{eV} / \AA$, respectively. PerdewWang functional ${ }^{28}$ known as PW91 was used in the generalized gradient approximation (GGA). In a previous study of ZGNRs, we showed that the GGA gives qualitatively the same results as hybrid density functional. ${ }^{14}$ Here, the calculated band gaps of the pristine ZGNR and BN nanoribbon with eight zigzag atom chains are 0.43 and $4.2 \mathrm{eV}$, respectively, in good agreement with previous DFT calculations. ${ }^{13,29}$

The Gibbs free energy of formation $\delta G$ (Refs. 15 and 16) for $\mathrm{C}_{i} \mathrm{BN}$ is defined as

$$
\delta G=E(\text { tot })-n_{\mathrm{H}} \mu_{\mathrm{H}}-n_{\mathrm{BN}} \mu_{\mathrm{BN}}-n_{\mathrm{C}} \mu_{\mathrm{C}}
$$

where $E$ (tot) is the cohesive energy per atom in $\mathrm{C}_{i} \mathrm{BN}$ ribbons, $n_{i}$ is the molar fraction of constituent $i(i=\mathrm{C}, \mathrm{H}$, and BN) in the ribbons, satisfying the relation $n_{\mathrm{H}}+n_{\mathrm{BN}}+n_{\mathrm{C}}=1$, and $\mu_{i}$ is the chemical potential of the constituent $i$ at a given state. We choose the binding energy per atom of $\mathrm{H}_{2}$ molecule as $\mu_{\mathrm{H}} . \mu_{\mathrm{C}}$ and $\mu_{\mathrm{BN}}$ are selected as the cohesive energies per atom of a single graphene sheet or BN sheet. Other choices of these reference values should not change the obtained qualitative picture.

The molecular dynamics simulations were performed with the general utility lattice program (GULP). ${ }^{30,31}$ The Dreiding force field ${ }^{32}$ was used to describe interaction between atoms. The $N V T$ ensemble was used with a temperature of $298 \mathrm{~K}$. A 1 ns trajectory with a 1 fs time step was obtained after $500 \mathrm{ps}$ of equilibration. (a)

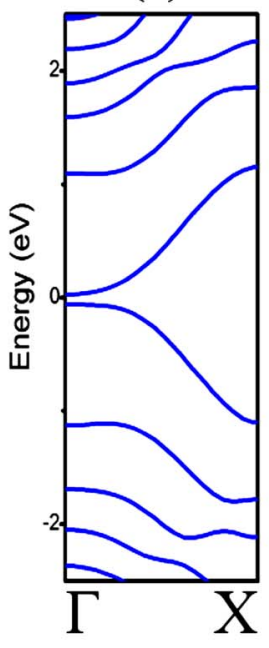

(b)

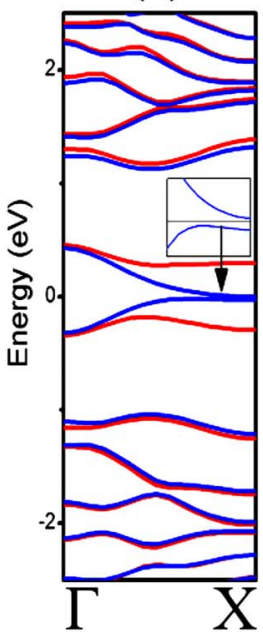

(c)

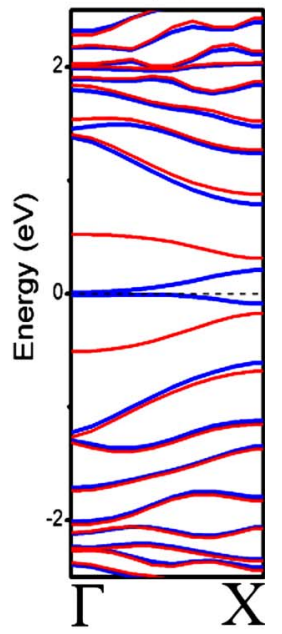

(d)

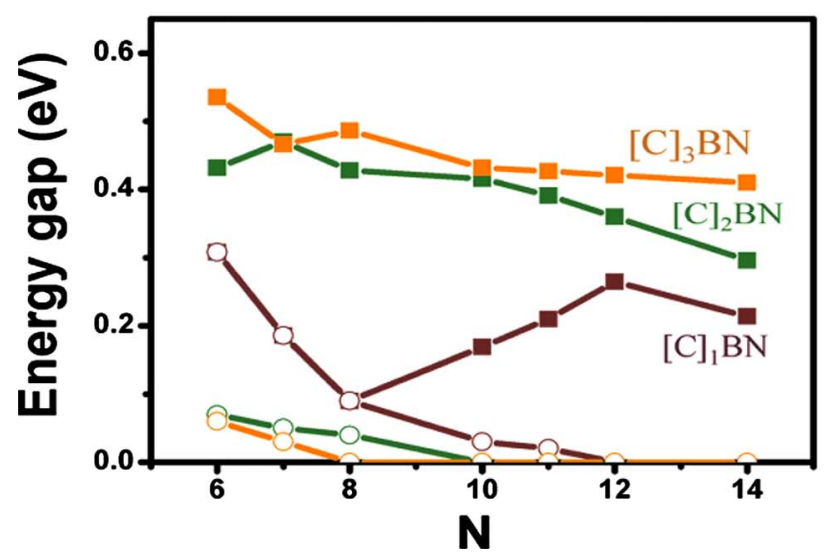

FIG. 2. (Color online) Band structures for (a) $\mathrm{C}_{1} \mathrm{BN}$, (b) $\mathrm{C}_{2} \mathrm{BN}$, and (c) $\mathrm{C}_{3} \mathrm{BN}$ with eight zigzag chains: The red lines represent the spin-up channel, while the blue ones mean spin-down. (d) The spin-resolved energy gaps $n-\mathrm{C}_{i} \mathrm{BN}$ nanoribbons. The filled square means spin-up channel, and the hollow circle represent spin-down channel.

\section{RESULTS AND DISCUSSION}

\section{A. Electronic structures}

The calculated electronic structures of $8-\mathrm{C}_{i} \mathrm{BN}$ $(i=1,2,3)$ are shown in Fig. 2. The band structures of $8-\mathrm{C}_{1} \mathrm{BN}$ and $8-\mathrm{C}_{2} \mathrm{BN}$ exhibit different patterns compared to $8-\mathrm{C}_{2} \mathrm{BN}$, which can be understood from the Brillouin-zone folding. As we know, for $8-\mathrm{C}_{1} \mathrm{BN}, 8-\mathrm{C}_{2} \mathrm{BN}$, and $8-\mathrm{C}_{3} \mathrm{BN}$ ribbons, which contain two, three, and four zigzag units, respectively. The Brillouin-zone becomes smaller for a larger unit cell. On the other hand, we also notice that the band structure differences between these three ribbons are not fully caused by the Brillouin-zone folding because of the different doping concentrations of $\mathrm{BN}$ chain in the $8-\mathrm{C}_{1} \mathrm{BN}$, $8-\mathrm{C}_{2} \mathrm{BN}$, and $8-\mathrm{C}_{3} \mathrm{BN}$ ribbons. Therefore, when $i$ is even, the largest gap locates at $\Gamma$ point, and when $i$ is odd the smallest gap locates at $\Gamma$ point. $8-\mathrm{C}_{1} \mathrm{BN}$ has a nonmagnetic ground state with a small band gap of $\sim 0.1 \mathrm{eV}$. Both $8-\mathrm{C}_{2} \mathrm{BN}$ and $8-\mathrm{C}_{3} \mathrm{BN}$ are spin polarized, and the latter is half metal since its VB and CB overlap at the Fermi level in the spin-down channel. This half-metallicity is unexpected, considering that 


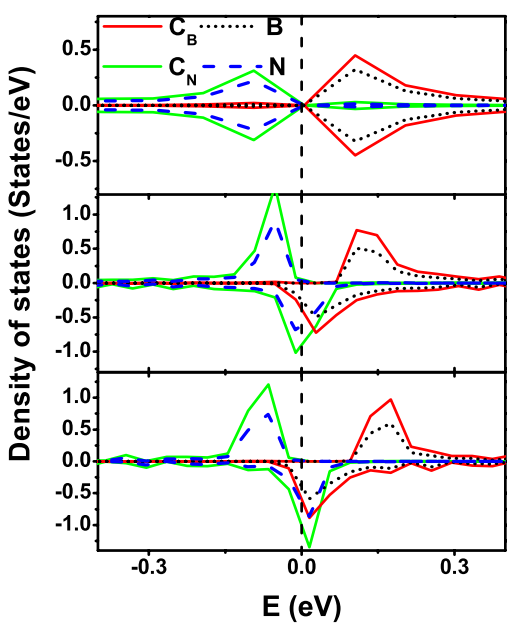

FIG. 3. (Color online) The project density of states for $\mathrm{C}_{1} \mathrm{BN}$ with (a) 8 , (b) 10 , and (c) 12 zigzag chains, respectively: $\mathrm{B}$ and $\mathrm{N}$ represent the relative edge atoms of the ribbons, while the $\mathrm{C}_{\mathrm{N}}$ and $\mathrm{C}_{\mathrm{B}}$ mean outmost carbon atoms at the $\mathrm{N}$ edge and the $\mathrm{B}$ edge, respectively. A Gaussian broadening with the variance $\sigma=0.02 \mathrm{eV}$ was applied.

both $\mathrm{C}$ and $\mathrm{BN}$ zigzag nanoribbons possess a finite band gap. We further examined nanoribbons with different widths $(n$ $=6-14)$. As shown in Fig. 2(d), all $\mathrm{C}_{i} \mathrm{BN}(\mathrm{i}=1,2,3)$ nanoribbons become half metal when $n \geq 12$. The critical widths are $n=12,10$, and 8 for $i=1,2$, and 3, respectively.

The calculated partial density of states indicates that the $8-\mathrm{C}_{1} \mathrm{BN}$ has no spin polarization as shown in Fig. 3. Near the Fermi level, the occupied states are contributed by $\mathrm{C}_{\mathrm{N}}$ and $\mathrm{N}$ edge atoms, while the unoccupied states are contributed by $\mathrm{C}_{\mathrm{B}}$ and $\mathrm{B}$ edge atoms. This is a charge polarized state with electron transfer from $C_{B}$ to $C_{N}$. The charge polarization is clearly seen in Fig. 4; the partial charge density from $0.05 \mathrm{eV}$ below the Fermi level to the Fermi level is localized at the $\mathrm{N}$-edge.

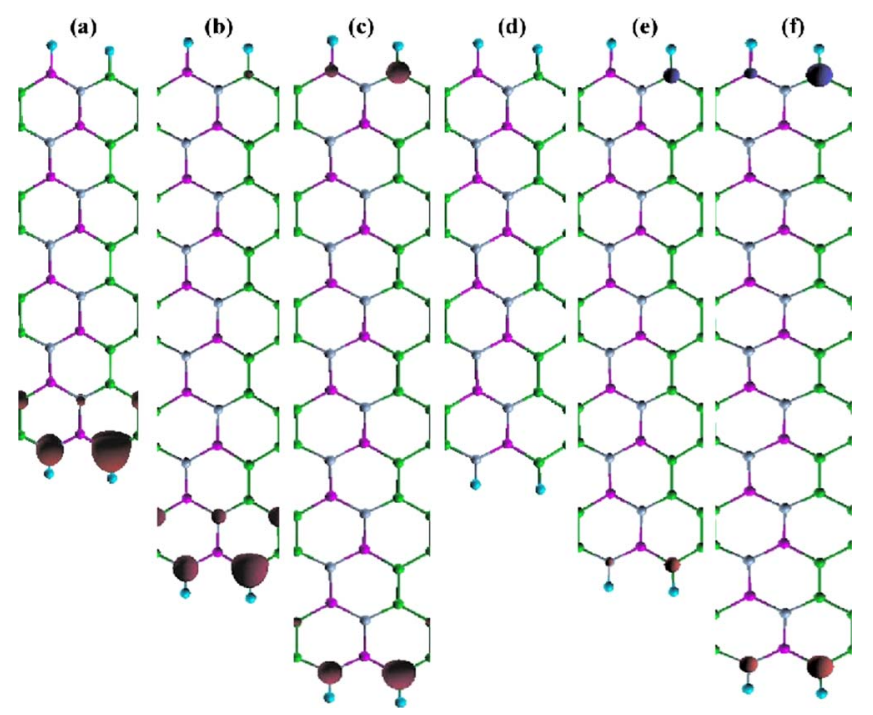

FIG. 4. (Color online) The partial charge density within energy range $\left(E_{f}\right.$ $-0.05 E_{f}$ ) in $\mathrm{eV}$ for $n$ - $\mathrm{C}_{1} \mathrm{BN}$ with $n$ equals (a) 8 , (b) 10 , and (c) 12: The isovalue is $0.02 \mathrm{e} / \AA^{3}$. Spin density of $n-\mathrm{C}_{1} \mathrm{BN}$ with $n$ equals (d) 8, (e) 10, and (f) 12 . The red and blue surfaces represent the spin-up and spin-down channels, respectively.
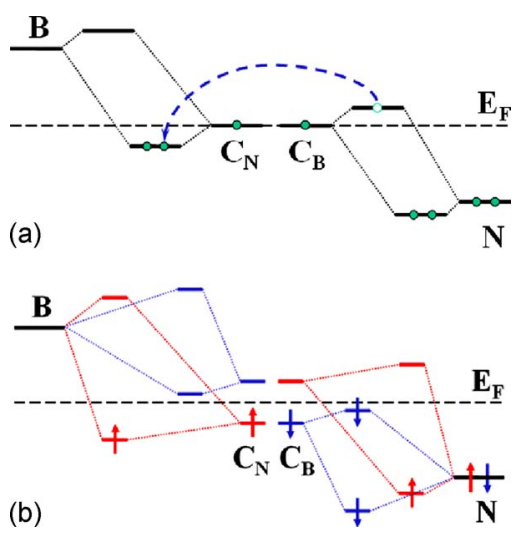

FIG. 5. (Color online) Schematic energy diagram for $\mathrm{C}_{i} \mathrm{BN}$ : (a) Charge polarization caused by $\pi$ electron hybridization between $\mathrm{C}$ and $\mathrm{BN}$. The blue arrow indicates the direction of electron transfer. (b) Competition between spin and charge polarization. Spin degenerate orbitals are represented by long level lines, and spin orbitals are denoted by short level lines. Red for the spin-up channel and blue for the spin-down channel.

\section{B. Competition between charge and spin polarizations}

In pristine ZGNR, every edge $\mathrm{C}$ atom at the edge has only two $\mathrm{C}$ neighbors and thus $1 / 3$ nonbonded extra $\pi$ electron, which gives rise to the edge states and a high electron density at the Fermi level. A stabilization mechanism is required to have a lower-energy ground state. ${ }^{19}$ In the chargepolarization mechanism, the extra electron transfers completely from one edge to the other. In the spin-polarization mechanism, the $\mathrm{C}$ atoms at the two opposing edges have spin-opposite electrons, which lowers the on-site Coulomb interaction $U$ between electrons with opposite spin at the same site. The spin mechanism is more favorable in energy. ${ }^{19}$ However, the charge polarized state can be further stabilized by an external electric field. Hence, a ZGNR can be changed from having spin polarized state to charge polarized state under external field. In either the spin or charge polarized state, a ZGNR is semiconductor. Here, the half-metallicity for ZGNR is found in the region where the two states compete with each other. ${ }^{14}$

The charge-ordered ground state of $8-\mathrm{C}_{1} \mathrm{BN}$ is stabilized by the mixing of $\pi$ orbitals of $\mathrm{C}$ and $\mathrm{BN}$. In $\mathrm{C}_{1} \mathrm{BN}$, each $\mathrm{C}_{\mathrm{B}}$ atom is covalently bonded to a $\mathrm{N}$ atom, while each $\mathrm{C}_{\mathrm{N}}$ atom is connected to a B atom. As shown in Fig. 5(a), without spin polarization, the occupied $\pi$ orbital of $\mathrm{N}$ is much lower in energy than the unoccupied $\pi$ orbital of B. The $\pi$ orbitals of $\mathrm{C}$ locate in the middle. The interaction between the $\pi$ orbitals of $\mathrm{C}_{\mathrm{B}}$ and $\mathrm{N}$ results in a higher antibonding orbital. However, the electron tends to occupy the lower bonding orbital between $C_{N}$ and $B$. Such an orbital interaction leads to electron transfer from $\mathrm{C}_{\mathrm{B}}$ to $\mathrm{C}_{\mathrm{N}}$.

As the nanoribbon width $(n)$ increases, the Coulomb interaction between the two edge states decreases, and thus the stabilization energy of the charge polarized state also decreases. On the other hand, the on-site Coulomb interaction is a local property, and the spin-polarization energy is independent of the ribbon width. ${ }^{19}$ Hence, the spin polarization becomes relatively more competitive as the nanoribbon width increases. 
As shown in Fig. 4(b), when the ribbon width is not wide enough (e.g., $n=10$ ), the ribbon still has an occupied $\mathrm{N}$-edge and nearly empty B-edge state. However, the spin degeneracy is already broken. The spin-up and spin-down electrons tend to occupy one of the two subsites of the honeycomb lattice, which leads to the energy of spin-down edge state higher than of the spin-up one. When $n=12$, the occupied spin-down state at the N-edge and the unoccupied spindown state at the B-edge cross over in energy. The B-edge is partly occupied, which weakens the charge polarization. More importantly, in this case, the spin-down channel becomes metallic. Therefore, the competition between the spin and charge polarization leads to half-metallicity in this hybrid-ribbon structure. As the ribbon width increases, a decrease in charge polarization [Figs. 4(a)-4(c)] and an increase in spin polarization [Figs. 4(d)-4(f)] can be clearly seen. The calculated total energies are also consistent with this conclusion. For the $8-\mathrm{C}_{1} \mathrm{BN}$, paramagnetic ordering is favorable. With the width increases to $n=10$, the antiferromagnetic state is almost degenerate with the paramagnetic one. For $n=12$, the antiferromagnetic state becomes the most stable one.

For $\mathrm{C}_{i} \mathrm{BN}$ with $i>1$, the $\pi$ orbital hybridization only occurs between $\mathrm{BN}$ and its nearest neighboring $\mathrm{C}$ atom row. In the $i=\infty$ limit, the electronic structure of $\mathrm{C}_{i} \mathrm{BN}$ will converge to that of ZGNR. For different $i$, we analyzed the competition between charge and spin polarization and the halfmetallicity based on the spin-polarized ZGNR ground state. As shown in Fig. 5(b), in ZGNR, C atoms at the two edges are degenerate in energy but with opposite spin. When the $\mathrm{BN}$ row is introduced, the energy of the bonding $\pi$ orbital between $C_{N}$ and $B$ is lower than that of the edge state in ZGNR. This bonding state is occupied by spin-up electrons. On the other hand, for $\mathrm{C}_{\mathrm{B}}$ and $\mathrm{N}$, both the bonding and antibonding states are occupied by the spin-down electrons. Hence, in $\mathrm{C}_{i} \mathrm{BN}$ without charge polarization, the VB maximum (VBM) stems from the antibonding states between $\mathrm{N}$ and $\mathrm{C}_{\mathrm{B}}$ in the spin-down channel, while the conducting band minimum (CBM) is composed of the bonding states between $B$ and $C_{N}$ in the same spin channel. With the increase in the ribbon width, the ZGNR band gap is reduced, and the VBM and CBM are closer in location. Once the VB and CB overlaps at a specific point ( $i=3$ and $n=8)$, spin-down electron at the B-edge will transfer to $\mathrm{N}$-edge. Consequently, at both edges, the spin-down channel is partially occupied, and the partial charge polarized nanoribbon becomes half metal.

\section{Stability}

The relative stability of the designed hybrid nanoribbons is very important for their practical use. To address this issue, we first calculate the Gibbs free energy of formation $\delta G$. The calculated $\delta G$ are $0.229,0.166$, and $0.126 \mathrm{eV} /$ atom for $12-\mathrm{C}_{1} \mathrm{BN}, 12-\mathrm{C}_{2} \mathrm{BN}$, and $12-\mathrm{C}_{3} \mathrm{BN}$, respectively. As a reference, $\delta G$ 's of $\mathrm{BN}$ nanoribbons and GNRs with $\mathrm{N}=12$ are 0.033 and $0.009 \mathrm{eV} /$ atom, respectively. The width dependence of $\delta G$ for $\mathrm{C}_{i} \mathrm{BN}$ is very week. Our results indicate that it is exothermic to form $\mathrm{C}_{i} \mathrm{BN}$ from $\mathrm{BN}$ nanoribbons and GNRs.

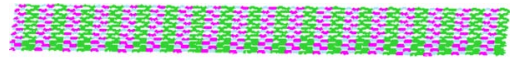

(a)

(b)

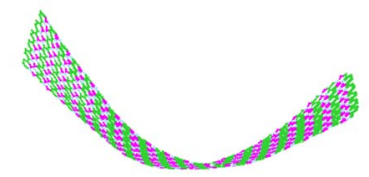

FIG. 6. (Color online) Two typical MD snapshots: (a) a unit cell of a hybrid $\mathrm{CBN}$ nanoribbon with periodic boundary condition along the ribbon direction: (b) a stand-alone hybrid CBN nanoribbon.

To study their stability under room temperature, molecular dynamics (MD) simulations for two $12-\mathrm{C}_{1} \mathrm{BN}$ systems have been performed. The first system is an infinite long CBN nanoribbon with periodic boundary condition along the ribbon direction. There are 936 atoms in a unit cell. The second system is a stand-alone CBN nanoribbon containing 1034 atoms. As shown in Fig. 6, in the 1 ns trajectory, the periodic system keeps the planner structure. For the standalone system, curling occurs, but the local structure is nearly planner. Therefore, we expect that the electronic structure will not be strongly affected. Our MD simulations thus suggest that such hybrid structures are stable at the room temperature.

Several methods have been reported to produce GNRs based on exfoliation, ${ }^{33}$ the radio-frequency plasma-enhanced chemical vapor deposition, ${ }^{34}$ epitaxial growth, ${ }^{35}$ or heat treatment of silicon carbide. ${ }^{36}$ It seems that our designed structures are difficult to be fabricated based on the above experimental skills. Fortunately, chemists have developed a chemical route to produce GNRs. ${ }^{37}$ The established bottom up approach ${ }^{38}$ provides a promise way to make our designed materials.

\section{SUMMARY}

In conclusion, we have studied spin-dependent electronic properties of heterogeneous $\mathrm{C} / \mathrm{BN}$ nanoribbons using DFT. Due to the competition between charge and spin polarizations, $n-\mathrm{C}_{i} \mathrm{BN}$ can be turned to half metal. In ZGNRs, spin polarization dominates. It has been suggested to apply external electric field to produce charge polarization thus halfmetallicity. However, high field intensity will hinder its electronics application. Here, the partial charge ordering is introduced in a chemical way. The $\pi$ orbital hybridization between $\mathrm{C}$ with $\mathrm{B}$ and $\mathrm{N}$, which have different chemical potentials, breaks the energetic symmetry of the two edge states. With suitable ribbon width $(n)$ and ratio between $\mathrm{C}$ and $\mathrm{BN}(i), n-\mathrm{C}_{i} \mathrm{BN}$ becomes half metal. MD simulation suggests that such hybrid structures are stable at the room temperature. Therefore, such metal-free half-metallicity may bring us the possibility to implement spintronic/electronic devices by bottom-up chemical synthetic approaches.

\section{ACKNOWLEDGMENTS}

This work is partially supported by the National Natural Science Foundation of China (Grant Nos. 5021091, 20533030, and 20628304), by National Key Basic Research Program under Grant No. 2006CB922004, by the USTC-HP 
HPC project, and by the SCCAS and Shanghai Supercomputer Center. The UNL group is also supported by U.S. DOE (Grant No. DE-FG02-04ER46164), NSF, and the Nebraska Research Initiative.

${ }^{1}$ H. W. Kroto, J. R. Heath, S. C. O’Brien, R. F. Curl, and R. E. Smalley, Nature (London) 318, 162 (1985).

${ }^{2}$ S. Iijima, Nature (London) 354, 56 (1991).

${ }^{3}$ T. L. Makarova, B. Sundqvist, R. Höhne, P. Esquinazi, Y. Kopelevich, P. Scharff, V. A. Davydov, L. S. Kashevarova, and A. V. Rakhmanina, Nature (London) 413, 716 (2001).

${ }^{4}$ P. Esquinazi, A. Setzer, R. Höhne, C. Semmelhack, Y. Kopelevich, D. Spemann, T. Butz, B. Kohlstrunk, and M. Löche, Phys. Rev. B 66, 024429 (2002).

${ }^{5}$ R. A. Wood, M. H. Lewis, M. R. Lees, S. M. Bennington, M. G. Cain, and N. Kitamura, J. Phys.: Condens. Matter 14, L385 (2002).

${ }^{6}$ J. Coey, M. Venkatesan, C. B. Fitzgerald, A. P. Douvalis, and I. S. Sanders, Nature (London) 420, 156 (2002).

${ }^{7}$ P. O. Lehtinen, A. S. Foster, A. Ayuela, A. Krasheninnikov, K. Nordlund, and R. Nieminen, Phys. Rev. Lett. 91, 017202 (2003).

${ }^{8}$ P. O. Lehtinen, A. S. Foster, A. Ayuela, T. T. Vehvilinen, and R. Nieminen, Phys. Rev. B 69, 155422 (2004).

${ }^{9}$ Y. C. Ma, P. O. Lehtinen, A. S. Foster, and R. Nieminen, New J. Phys. 6, 68 (2004).

${ }^{10}$ K. S. Novoselov, A. K. Geim, S. V. Morozov, D. Jiang, M. I. Katsnelson, I. V. Grigorieva, S. V. Dubonos, and A. A. Firsov, Nature (London) 438 197 (2005).

${ }^{11}$ Y. B. Zhang, Y. W. Tan, H. L. Stormer, and P. Kim, Nature (London) 438, 201 (2005).

${ }^{12}$ C. Berger, Z. M. Song, X. B. Li, X. S. Wu, N. Brown, C. Naud, D. Mayou, T. B. Li, J. Hass, A. N. Marchenkov, E. H. Conrad, P. N. First, and W. A. de Heer, Science 312, 1191 (2006).

${ }^{13}$ Y.-W. Son, M. L. Cohen, and S. G. Louie, Nature (London) 444, 347 (2006).

${ }^{14}$ E.-J. Kan, Z. Y. Li, J. L. Yang, and J. G. Hou, Appl. Phys. Lett. 91, 243116 (2007).

${ }^{15}$ O. Hod, V. Barone, J. Peralta, and G. Scuseria, Nano Lett. 7, 2295 (2007).
${ }^{16}$ E.-J. Kan, Z. Y. Li, J. L. Yang, and J. G. Hou, J. Am. Chem. Soc. 130, 4224 (2008)

${ }^{17}$ K. Kusakabe and M. Maruyama, Phys. Rev. B 67, 092406 (2003).

${ }^{18}$ H. Lee, Y.-W. Son, N. Park, S. Han, and J. Yu, Phys. Rev. B 72, 174431 (2005).

${ }^{19}$ L. Pisani, J. A. Chan, B. Montanari, and N. Harrison, Phys. Rev. B 75, 064418 (2007).

${ }^{20}$ V. Barone, O. Hod, and G. Scuseria, Nano Lett. 6, 2748 (2006).

${ }^{21}$ M. Fujita, K. Wakabayashi, K. Nakada, and K. Kusakabe, J. Phys. Soc. Jpn. 65, 1920 (1996).

${ }^{22}$ K. Nakada, M. Fujita, G. Dresselhaus, and M. Dresselhaus, Phys. Rev. B 54, 17954 (1996).

${ }^{23}$ K. Wakabayashi, M. Sigrist, and M. Fujita, J. Phys. Soc. Jpn. 67, 2089 (1998).

${ }^{24}$ K. Wakabayashi, M. Fujita, H. Ajiki, and M. Sigrist, Phys. Rev. B 59, 8271 (1999)

${ }^{25}$ G. Kresse and J. Hafner, Phys. Rev. B 47, 558 (1993).

${ }^{26}$ P. E. Blochl, Phys. Rev. B 50, 17953 (1994).

${ }^{27}$ G. Kresse and D. Joubert, Phys. Rev. B 59, 1758 (1999).

${ }^{28}$ J. P. Perdew and Y. Wang, Phys. Rev. B 45, 13244 (1992).

${ }^{29}$ J. Nakamura, T. Nitta, and A. Natori, Phys. Rev. B 72, 205429 (2005).

${ }^{30}$ D. Gale, J. Chem. Soc., Faraday Trans. 93, 629 (1997).

${ }^{31}$ D. Gale and A. L. Rohl, Mol. Simul. 29, 291 (2003).

${ }^{32}$ S. L. Mayo, B. D. Olafson, and W. A. Goddard III, J. Phys. Chem. 94, 8897 (1990)

${ }^{33}$ K. S. Novoselov, A. K. Geim, S. V. Morozov, D. Jiang, Y. Zhang, S. V. Dubonos, I. V. Grigorieva, and A. A. Firsov, Science 306, 666 (2004).

${ }^{34}$ J. Wang, M. Zhu, R. A. Outlaw, X. L. Zhao, D. Manos, B. Holloway, and V. Mammana, Appl. Phys. Lett. 85, 1265 (2004).

${ }^{35}$ C. Berger, Z. Song, X. Li, X. Wu, N. Brown, C. Naud, D. Mayou, T. Li, J. Hass, A. Marchenkov, E. Conrad, P. First, and W. De Heer, Science 312, 1191 (2006)

${ }^{36}$ J. Hass, R. Feng, T. Li, X. Li, Z. Zong, W. de Heer, P. First, E. Conrad, C. Jeffrey, and C. Berger, Appl. Phys. Lett. 89, 143106 (2006).

${ }^{37}$ X. Li, X. Wang, L. Zhang, S. Lee, and H. Dai, Science 319, 1229 (2008).

${ }^{38}$ X. Yang, X. Dou, A. Rouhanipour, L. Zhi, H. Räder, and K. Müllen, J. Am. Chem. Soc. 130, 4216 (2008). 